\title{
On the $\Psi$ - Conditional Stability of Nonlinear Lyapunov Matrix Differential Equations
}

Aurel Diamandescu

\begin{abstract}
It is proved (necessary and) sufficient conditions for $\Psi$ - conditional stability of the trivial solution of linear or nonlinear Lyapunov matrix differential equations.
\end{abstract}

AMS Subject Classification (2000). 34D20, 34D05.

Keywords. $\Psi$ - conditional stability, $\Psi$ - instability, Lyapunov matrix differential equation.

\section{Introduction}

The Lyapunov matrix differential equations occur in many branches of control theory such as optimal control and stability analysis..

Recent works for $\Psi$ - boundedness, $\Psi$ - stability, $\Psi$ - instability, controllability, dichotomy and conditioning for Lyapunov matrix differential equations have been given in many papers. See, for example, $[5-12]$ and the references cited therein.

The purpose of present paper is to prove (necessary and) sufficient conditions for $\Psi$ - conditional stability of trivial solution of the nonlinear Lyapunov matrix differential equation

$$
Z^{\prime}=A(t) Z+Z B(t)+F(t, Z)
$$

and the linear Lyapunov matrix differential equation

$$
Z^{\prime}=\left[A(t)+A_{1}(t)\right] Z+Z\left[B(t)+B_{1}(t)\right],
$$


which can be seen as a perturbed equations of the linear equation

$$
Z^{\prime}=A(t) Z+Z B(t)
$$

We investigate conditions on the fundamental matrices of the equations

$$
\begin{aligned}
& Z^{\prime}=A(t) Z, \\
& Z^{\prime}=Z B(t)
\end{aligned}
$$

and on the functions $A_{1}, B_{1}$ and $F$ under which the trivial solutions of the equations (1.1), (1.2) and (1.3) are $\Psi$ - conditionally stable on $\mathbb{R}_{+}$. Here, $\Psi$ is a matrix function whose introduction permits us obtaining a mixed asymptotic behavior for the components of solutions.

The main tool used in this paper is the technique of Kronecker product of matrices, which has been successfully applied in various fields of matrix theory, group theory and particle physics. See, for example, the above cited papers and the references cited therein.

\section{Preliminaries}

In this section we present some basic definitions, notations, hypotheses and results which are useful later on.

Let $\mathbb{R}^{n}$ be the Euclidean $\mathrm{n}$-dimensional space. For $\mathrm{x}=\left(\mathrm{x}_{1}, \mathrm{x}_{2}, \mathrm{x}_{3}, \ldots, \mathrm{x}_{\mathrm{n}}\right)^{\mathrm{T}} \in$ $\mathbb{R}^{\mathrm{n}}$, let $\|\mathrm{x}\|=\max \left\{\left|\mathrm{x}_{1}\right|,\left|\mathrm{x}_{2}\right|,\left|\mathrm{x}_{3}\right|, \ldots,\left|\mathrm{x}_{\mathrm{n}}\right|\right\}$ be the norm of $\mathrm{x}$ ( ${ }^{\mathrm{T}}$ denotes transpose).

Let $\mathbb{M}_{m \times n}$ be the linear space of all $m \times n$ real valued matrices.

For a matrix $\mathrm{A}=\left(\mathrm{a}_{\mathrm{ij}}\right) \in \mathbb{M}_{\mathrm{n} \times \mathrm{n}}$, we define the norm $|\mathrm{A}|$ by $|\mathrm{A}|=\sup _{\|\mathrm{x}\| \leq 1}\|\mathrm{Ax}\|$.

It is well-known that $|\mathrm{A}|=\max _{1 \leq \mathrm{i} \leq \mathrm{n}}\left\{\sum_{\mathrm{j}=1}^{\mathrm{n}}\left|\mathrm{a}_{\mathrm{ij}}\right|\right\}$.

By a solution of the equation (1.1) we mean a continuous differentiable $d \times d$ matrix function satisfying the equation (1.1) for all $t \in \mathbb{R}_{+}$.

In equation (1.3), we assume that $\mathrm{A}$ and $\mathrm{B}$ are continuous $d \times d$ matrices on $\mathbb{R}_{+}=[0, \infty)$. It is well-known that continuity of $A$ and $B$ ensure the existence and uniqueness on $\mathbb{R}_{+}$of a solution of (1.3) passing through any given point $\left(t_{0}, Z_{0}\right) \in \mathbb{R}_{+} \times \mathbb{M}_{d \times d}$.

In addition, in equation (1.1), we assume that $F: \mathbb{R}_{+} \times \mathbb{M}_{d \times d} \longrightarrow \mathbb{M}_{d \times d}$ is continuous such that $F\left(t, O_{d}\right)=O_{d}$ (null matrix of order $d \times d$ ).

It is well-known that these conditions ensure the local existence of a solution passing through any given point $\left(t_{0}, Z_{0}\right) \in \mathbb{R}_{+} \times \mathbb{M}_{d \times d}$, but it not guarantee that the solution is unique or that it can be continued for large values of $t$. 
Let $\Psi_{i}: \mathbb{R}_{+} \longrightarrow(0, \infty), \mathrm{i}=1,2, \ldots, \mathrm{n}$, be continuous functions and

$$
\Psi=\operatorname{diag}\left[\Psi_{1}, \Psi_{2}, \cdots \Psi_{n}\right]
$$

Def inition 2.1. ([3], [6]). The solution $z(t)$ of the differential equation $z^{\prime}$ $=f(t, z)$ (where $z \in R^{d}$ and $f$ is a continuous $d$ vector function) is said to be $\Psi$ - stable on $R_{+}$if for every $\varepsilon>0$ and every $t_{0} \in \mathbb{R}_{+}$, there exists a $\delta=\delta\left(\varepsilon, t_{0}\right)>0$ such that, any solution $\widetilde{\mathrm{z}}(t)$ of the equation which satisfies the inequality $\left\|\Psi\left(t_{0}\right)\left(\widetilde{\mathrm{z}}\left(t_{0}\right)-z\left(t_{0}\right)\right)\right\|<\delta$, exists and satisfies the inequality $\|\Psi(t)(\widetilde{\mathrm{z}}(t)-z(t))\|<\varepsilon$ for all $t \geq t_{0}$.

Otherwise, is said that the solution $z(t)$ is $\Psi$ - unstable on $R_{+}$.

Def inition 2.2. ([3]). A function $\varphi: R_{+} \longrightarrow R^{d}$ is said to be $\Psi-$ bounded on $R_{+}$if $\Psi(t) \varphi(t)$ is bounded on $R_{+}$.

Otherwise, is said that the function $\varphi$ is $\Psi$ - unbounded on $R_{+}$.

Def inition 2.3. ([3]). The solution $z(t)$ of the differential equation $z^{\prime}=$ $f(t, z)$ is said to be $\Psi$ - conditionally stable on $R_{+}$if it is not $\Psi$ - stable on $R_{+}$but there exists a sequence $\left(z_{n}(t)\right)$ of solutions of the equation defined for all $t \in R_{+}$such that

$$
\lim _{n \rightarrow \infty} \Psi(t) z_{n}(t)=\Psi(t) z(t) \text {, uniformly on } R_{+} .
$$

Remark 2.1. These definitions generalize the classical definitions of various types of stability (see [2]).

Now, we extend these definitions for a matrix differential equation $Z^{\prime}=$ $F(t, Z)$, where $Z \in \mathbb{M}_{d \times d}$ and $F: \mathbb{R}_{+} \times \mathbb{M}_{d \times d} \longrightarrow \mathbb{M}_{d \times d}$ is a continuous function.

Def inition 2.4. ([6]). The solution $Z(t)$ of the matrix differential equation $Z^{\prime}=F(t, Z)$ is said to be $\Psi$ - stable on $R_{+}$if for every $\varepsilon>0$ and every $t_{0} \in \mathbb{R}_{+}$, there exists a $\delta=\delta\left(\varepsilon, t_{0}\right)>0$ such that, any solution $\widetilde{\mathrm{Z}}(t)$ of the equation which satisfies the inequality $\left|\Psi\left(t_{0}\right)\left(\widetilde{Z}\left(t_{0}\right)-Z\left(t_{0}\right)\right)\right|<\delta$, exists and satisfies the inequality $|\Psi(t)(\widetilde{Z}(t)-Z(t))|<\varepsilon$ for all $t \geq t_{0}$. Otherwise, is said that the solution $Z(t)$ is $\Psi$ - unstable on $R_{+}$.

Def inition 2.5. A matrix function $M: \mathbb{R}_{+} \longrightarrow \mathbb{M}_{d \times d}$ is said to be $\Psi-$ bounded on $R_{+}$if the matrix function $\Psi(t) M(t)$ is bounded on $R_{+}$(i.e. there exists $m>0$ such that $|\Psi(t) M(t)| \leq m$, for all $\left.t \in R_{+}\right)$.

Otherwise, is said that the function $M$ is $\Psi$ - unbounded on $R_{+}$. 
Def inition 2.6. The solution $Z(t)$ of the matrix differential equation $Z^{\prime}=$ $F(t, Z)$ is said to be $\Psi$ - conditionally stable on $R_{+}$if it is not $\Psi$ - stable on $R_{+}$but there exists a sequence $\left(Z_{n}(t)\right)$ of solutions of the equation defined on $R_{+}$such that

$$
\lim _{n \rightarrow \infty} \Psi(t) Z_{n}(t)=\Psi(t) Z(t) \text {, uniformly on } R_{+} .
$$

Remark 2.2. 1. It is easy to see that if $\Psi(t)$ and $\Psi^{-1}(t)$ are bounded on $\mathbb{R}_{+}$, then the $\Psi$ - stability and $\Psi$ - conditional stability are equivalent with the classical stability and conditional stability respectively.

2. In the same manner as in classical conditional stability, we can speak about $\Psi$ - conditional stability of a linear matrix differential equation (1.3), (1.4) or (1.5).

Indeed, let $\mathrm{X}(\mathrm{t}), \mathrm{Y}(\mathrm{t})$ be two solutions of the equation (1.4). Suppose that the solution $\mathrm{X}(\mathrm{t})$ is $\Psi$ - conditionally stable on $\mathbb{R}_{+}$. From Definition 2.6, $\mathrm{X}(\mathrm{t})$ is not $\Psi$ - stable on $\mathbb{R}_{+}$and there exists a sequence $\left(\mathrm{X}_{n}(\mathrm{t})\right)$ of solutions of the equation defined on $\mathbb{R}_{+}$such that $\lim _{n \rightarrow \infty} \Psi(t) X_{n}(t)=\Psi(t) X(t)$, uniformly on $\mathbb{R}_{+}$. Then, $\mathrm{Y}(\mathrm{t})$ is not $\Psi$ - stable on $\mathbb{R}_{+}$(see Theorem 1 , [5]) and $\lim _{n \rightarrow \infty} \Psi(t) Y_{n}(t)=\Psi(t) Y(t)$, uniformly on $\mathbb{R}_{+}$, where

$$
Y_{n}(t)=X_{n}(t)-X(t)+Y(t), t \in \mathbb{R}_{+}, n \in \mathbb{N},
$$

are solutions of (1.4). Thus, all solutions of (1.4) are $\Psi$ - conditionally stable on $\mathbb{R}_{+}$.

The last cases are similar.

Def inition 2.7. ([1]). Let $A=\left(a_{i j}\right) \in M_{m \times n}$ and $B=\left(b_{i j}\right) \in M_{p \times q}$. The Kronecker product of $A$ and $B$, written $A \otimes B$, is def ined to be the partitioned matrix

$$
A \otimes B=\left(\begin{array}{cccc}
a_{11} B & a_{12} B & \cdots & a_{1 n} B \\
a_{21} B & a_{22} B & \cdots & a_{2 n} B \\
\vdots & \vdots & \vdots & \vdots \\
a_{m 1} B & a_{m 2} B & \cdots & a_{m n} B
\end{array}\right) .
$$

Obviously, $A \otimes B \in \mathbb{M}_{m p \times n q}$.

The important rules of calculation of the Kronecker product there are in next Lemma.

Lemma 2.1. ([1]). The Kronecker product has the following properties and rules, provided that the dimension of the matrices are such that the various expressions exist:

1). $A \otimes(B \otimes C)=(A \otimes B) \otimes C$; 
2). $(A \otimes B)^{\mathrm{T}}=A^{\mathrm{T}} \otimes B^{\mathrm{T}}$;

3). $(A \otimes B) \cdot(C \otimes D)=(A \cdot C) \otimes(B \cdot D)$;

4). $(A \otimes B)^{-1}=A^{-1} \otimes B^{-1}$

5). $A \otimes(B+C)=A \otimes B+A \otimes C$;

6). $(A+B) \otimes C=A \otimes C+B \otimes C$;

7). $I_{\mathrm{p}} \otimes A=\left(\begin{array}{cccc}\mathrm{A} & \mathrm{O} & \cdots & \mathrm{O} \\ \mathrm{O} & \mathrm{A} & \cdots & \mathrm{O} \\ \vdots & \vdots & \vdots & \vdots \\ \mathrm{O} & \mathrm{O} & \cdots & \mathrm{A}\end{array}\right)$;

8). $(A(t) \otimes B(t))^{\prime}=A^{\prime}(t) \otimes B(t)+A(t) \otimes B^{\prime}(t)$; (' denotes the derivative $\left.\frac{d}{d t}\right)$.

Proof. See in [1].

Def inition 2.8. The application Vec: $\mathbb{M}_{m \times n} \longrightarrow \mathbb{R}^{m n}$, defined by

$$
\operatorname{Vec}(A)=\left(a_{11}, a_{21}, \cdots, a_{m 1}, a_{12}, a_{22}, \cdots, a_{m 2}, \cdots, a_{1 n}, a_{2 n}, \cdots, a_{m n}\right)^{T},
$$

where $A=\left(a_{i j}\right) \in \mathbb{M}_{m \times n}$, is called the vectorization operator.

Lemma 2.2. ([5]). The vectorization operator

$$
\operatorname{Vec}: \mathbb{M}_{m \times n} \longrightarrow \mathbb{R}^{m n}, A \longrightarrow \operatorname{Vec}(A)
$$

is a linear and one-to-one operator. In addition, $V e c$ and $V e c^{-1}$ are continuous operators.

Proof. See Lemma 2, [5].

Lemma 2.3. A function $F: \mathbb{R}_{+} \longrightarrow \mathbb{M}_{n \times n}$ is a continuous (differentiable) matrix function on $\mathbb{R}_{+}$if and only if the function $f: \mathbb{R}_{+} \longrightarrow \mathbb{R}^{n^{2}}$, def ined by $f(t)=\operatorname{Vec}(F(t))$, is a continuous (differentiable) vector function on $\mathbb{R}_{+}$. Proof. It is a simple exercise.

We recall that the vectorization operator Vec has the following properties as concerns the calculations.

Lemma 2.4. If $A, B, M \in \mathbb{M}_{n \times n}$, then

1). $\operatorname{Vec}(A M B)=\left(B^{T} \otimes A\right) \cdot \operatorname{Vec}(M)$;

2). $\operatorname{Vec}(M B)=\left(B^{T} \otimes I_{n}\right) \cdot \operatorname{Vec}(M)$;

3). $\operatorname{Vec}(A M)=\left(I_{n} \otimes A\right) \cdot \operatorname{Vec}(M)$;

4). $\operatorname{Vec}(A M)=\left(M^{T} \otimes A\right) \cdot \operatorname{Vec}\left(I_{n}\right)$.

Proof. It is a simple exercise.

The following lemmas play a vital role in the proofs of main results of present paper. 
Lemma 2.5. ([5]). The matrix function $Z(t)$ is a solution of (1.1) on $\mathbb{R}_{+}$if and only if the vector function $z(t)=\operatorname{Vec}(Z(t))$ is a solution of the differential system

$$
z^{\prime}=\left(I_{d} \otimes A(t)+B^{T}(t) \otimes I_{d}\right) z+f(t, z)
$$

where $f(t, z)=\operatorname{Vec}(F(t, Z))$, on the same interval $\mathbb{R}_{+}$.

Proof. See Lemma 5, [5].

Def inition 2.9. The above system (2.1) is called "corresponding Kronecker product system associated with (1.1)".

Lemma 2.6. ([5]). For every matrix function $M: \mathbb{R}_{+} \longrightarrow \mathbb{M}_{d \times d}$,

$$
\frac{1}{d}|\Psi(t) M(t)| \leq\left\|\left(I_{d} \otimes \Psi(t)\right) \operatorname{Vec}(M(t))\right\|_{\mathbb{R}^{d^{2}}} \leq|\Psi(t) M(t)|, t \geq 0 .
$$

Proof. See Lemma 6, [5].

Lemma 2.7. The trivial solution of the equation (1.1) is $\Psi$ - conditionally stable on $R_{+}$if and only if the trivial solution of the corresponding Kronecker product system (2.1) is $I_{d} \otimes \Psi-$ conditionally stable on $R_{+}$.

Proof. First, suppose that the trivial solution of the equation (1.1) is $\Psi-$ conditionally stable on $\mathbb{R}_{+}$. According to Definition 2.6, this solution is not $\Psi$ - stable on $\mathbb{R}_{+}$but there exists a sequence $\left(Z_{n}(t)\right)$ of solutions of the equation defined on $\mathbb{R}_{+}$such that

$$
\lim _{n \rightarrow \infty} \Psi(t) Z_{n}(t)=O_{d} \text {, uniformly on } R_{+} \text {. }
$$

From Lemma 2.5 and Lemma 7, [6], the trivial solution of (2.1) is not $I_{d} \otimes \Psi-$ stable on $\mathbb{R}_{+}$. In addition, from the inequality (2.2), we have that

$$
\left\|\left(I_{d} \otimes \Psi(t)\right) \operatorname{Vec}\left(Z_{n}(t)\right)\right\|_{\mathbb{R}^{d^{2}}} \leq\left|\Psi(t) Z_{n}(t)\right|, t \geq 0 .
$$

It follows that

$$
\lim _{n \rightarrow \infty}\left(I_{d} \otimes \Psi(t)\right) \operatorname{Vec}\left(Z_{n}(t)\right)=0 \text {, uniformly on } \mathbb{R}_{+} .
$$

From these results, Lemma 2.5 and Definition 2.3, it follows that the trivial solution of (2.1) is $I_{d} \otimes \Psi$ - conditionally stable on $\mathbb{R}_{+}$.

Suppose, conversely, that the trivial solution of (2.1) is $I_{d} \otimes \Psi-$ conditionally stable on $\mathbb{R}_{+}$. According to Definition 2.3, this solution is not $I_{d} \otimes \Psi-$ stable on $\mathbb{R}_{+}$but there exists a sequence $\left(\mathrm{z}_{n}(\mathrm{t})\right)$ of solutions of the system $(2.1)$ defined on $\mathbb{R}_{+}$such that 
$\lim _{n \rightarrow \infty}\left(I_{d} \otimes \Psi(t)\right) z_{n}(t)=0$, uniformly on $\mathbb{R}_{+}$.

From Lemma 2.5 and Lemma 7, [6], the trivial solution of (1.1) is not $\Psi-$ stable on $\mathbb{R}_{+}$. In addition, from the inequality (2.2), we have that

$$
\frac{1}{d}\left|\Psi(t) \mathcal{V} e c^{-1}\left(z_{n}(t)\right)\right| \leq\left\|\left(I_{d} \otimes \Psi(t)\right) z_{n}(t)\right\|_{\mathbb{R}^{d^{2}}} .
$$

It follows that

$$
\lim _{n \rightarrow \infty} \Psi(t) \mathcal{V} e c^{-1}\left(z_{n}(t)\right)=O_{d}, \text { uniformly on } R_{+} .
$$

(here, $\left(\mathcal{V e c}^{-1}\left(z_{n}(t)\right)\right)$ is a sequence of solutions of the equation (1.1)).

From these results, Lemma 2.5 and Definition 2.6, it follows that the trivial solution of $(1.1)$ is $\Psi$ - conditionally stable on $\mathbb{R}_{+}$.

The proof is now complete.

Lemma 2.8. ([5]). Let $X(t)$ and $Y(t)$ be a fundamental matrices for the equations (1.4) and (1.5) respectively.

Then, the matrix $Z(t)=Y^{\mathrm{T}}(t) \otimes X(t)$ is a fundamental matrix for the corresponding Kronecker product system associated with (1.3), i.e. for the differential system

$$
z^{\prime}=\left(I_{d} \otimes A(t)+B^{T}(t) \otimes I_{d}\right) z
$$

Proof. See Lemma 9, [5].

\section{$3 \quad \Psi$ - conditional stability of linear matrix differential equations}

The purpose of this section is to study the $\Psi$ - conditional stability of the linear matrix differential equations (1.4) and

$$
Z^{\prime}=\left(A(t)+A_{1}(t)\right) Z
$$

The conditions for $\Psi$ - conditional stability of the linear matrix differential equation (1.4) can be expressed in terms of solutions or in terms of a fundamental matrix for (1.4).

Theorem 3.1. The linear matrix differential equation (1.4) is $\Psi$ - conditionally stable on $\mathbb{R}_{+}$if and only if it has a $\Psi$ - unbounded solution on $\mathbb{R}_{+}$ and a nontrivial $\Psi$ - bounded solution on $\mathbb{R}_{+}$. 
Proof. First, we shall prove the "only if" part.

Suppose that the linear matrix differential equation (1.4) is $\Psi$ - conditionally stable on $\mathbb{R}_{+}$. Let $\mathrm{X}(\mathrm{t})$ be a fundamental matrix for (1.4). From the above Definition 2.6, Remark 2.2 and Theorem 1, [6], it follows that $|\Psi(t) X(t)|$ is unbounded on $\mathbb{R}_{+}$. Thus, the linear equation (1.4) has at least one $\Psi-$ unbounded solution on $\mathbb{R}_{+}$. In addition, there exists a sequence $\left(\mathrm{X}_{n}(\mathrm{t})\right)$ of nontrivial solutions of (1.4) such that

$$
\lim _{n \rightarrow \infty} \Psi(t) X_{n}(t)=O_{d}, \text { uniformly on } R_{+} .
$$

Thus, there exist the constant matrices $C_{n} \neq O_{d}$ such that

$$
\lim _{n \rightarrow \infty} \Psi(t) X(t) C_{n}=O_{d} \text {, uniformly on } R_{+} \text {. }
$$

Hence, there exists $n_{0} \in \mathbb{N}$ such that $\left|\Psi(t) X(t) C_{n}\right|<1$ for $n \geq n_{0}$ and $t \in \mathbb{R}_{+}$. We choose $C_{m} \neq O_{d}, m \geq n_{0}$. Thus, $X(t) C_{m}$ is a $\Psi$ - bounded nontrivial solution of (1.4) on $\mathbb{R}_{+}$.

Now, we shall prove the "if" part.

Suppose that the linear matrix differential equation (1.4) has a $\Psi$ - unbounded solution on $\mathbb{R}_{+}$and a nontrivial solution $\mathrm{X}_{0}(\mathrm{t})$ such that $\Psi(t) X_{0}(t)$ is bounded on $\mathbb{R}_{+}$. It follows that the fundamental matrix $\mathrm{X}(\mathrm{t})$ for (1.4) is such that $|\Psi(t) X(t)|$ is unbounded on $\mathbb{R}_{+}$. Consequently, the linear matrix differential equation (1.4) is $\Psi$ - unstable on $\mathbb{R}_{+}$(see Theorem $1,[6]$ ). On the other hand, $\left(\frac{1}{n} X_{0}(t)\right)$ is a sequence of solutions of (1.4) such that

$$
\lim _{n \rightarrow \infty} \Psi(t)\left(\frac{1}{n} X_{0}(t)\right)=O_{d} \text {, uniformly on } R_{+} \text {. }
$$

Thus, the linear matrix differential equation (1.4) is $\Psi$ - conditionally stable on $\mathbb{R}_{+}$.

The proof is now complete.

Theorem 3.2. Let $\mathrm{X}(\mathrm{t})$ be a fundamental matrix for the linear matrix differential equation (1.4).

Then, the linear matrix differential equation (1.4) is $\Psi$ - conditionally stable on $\mathbb{R}_{+}$if and only if the following conditions are true:

a). there exists a projections $\mathrm{P}_{1}$ such that $\Psi(\mathrm{t}) \mathrm{X}(\mathrm{t}) \mathrm{P}_{1}$ is unbounded on $\mathbb{R}_{+}$; b). there exists a projections $\mathrm{P}_{2} \neq 0$ such that $\Psi(\mathrm{t}) \mathrm{X}(\mathrm{t}) \mathrm{P}_{2}$ is bounded on $\mathbb{R}_{+}$.

Proof. First, we shall prove the "only if" part.

From $\Psi$ - conditional stability on $\mathbb{R}_{+}$of (1.4) and Theorem 1, [5], it follows that there exists a projections $\mathrm{P}_{1}$ such that $\Psi(\mathrm{t}) \mathrm{X}(\mathrm{t}) \mathrm{P}_{1}$ is unbounded on $\mathbb{R}_{+}$. 
In addition, from the above Theorem, there exists a matrix $C \neq O_{d}$ such that $\mathrm{X}(\mathrm{t}) \mathrm{C}$ is a $\Psi$ - bounded nontrivial solution of (1.4) on $\mathbb{R}_{+}$. Let the column $\mathrm{c}_{i}$ $=\left(\mathrm{c}_{1 i}, \mathrm{c}_{2 i}, \ldots, \mathrm{c}_{d i}\right)^{T} \neq 0$ of $\mathrm{C}$. Let $\mathrm{c}_{j i}=\left\|c_{i}\right\|$. Let $\mathrm{P}_{2}$ be the nul matrix $O_{d}$ in which the $\mathrm{j}$ column is replaced with the column $\mathrm{c}_{j i}^{-1} c_{i}$. It is easy to see that $\mathrm{P}_{2} \neq 0$ is a projection and $\Psi(\mathrm{t}) \mathrm{X}(\mathrm{t}) \mathrm{P}_{2}$ is bounded on $\mathbb{R}_{+}$.

Now, we shall prove the "if" part.

From the hypothesis a) and Theorem 1, [5], it follows that the linear matrix differential equation (1.4) is $\Psi$ - unstable on $\mathbb{R}_{+}$.

Let $\mathrm{X}_{0}(\mathrm{t})$ be a nontrivial solution on $\mathbb{R}_{+}$of the linear matrix differential equation (1.4). Let $\left(\lambda_{n}\right)$ be such that $\lambda_{n} \in \mathbb{R} \backslash\{1\}, \lim _{n \rightarrow \infty} \lambda_{n}=1$ and let $\left(\mathrm{X}_{n}(\mathrm{t})\right)$ be defined by

$$
X_{n}(t)=X(t) P_{2} X^{-1}(0)\left(\lambda_{n} X_{0}(0)\right)+X(t)\left(I-P_{2}\right) X^{-1}(0) X_{0}(0), t \geq 0 .
$$

It is easy to see that $X_{n}(t)$ are solutions of the linear matrix differential equation (1.4). For $n \in \mathbb{N}$ and $t \geq 0$, we have

$$
\begin{aligned}
& \left|\Psi(t) X_{n}(t)-\Psi(t) X_{0}(t)\right|= \\
& =\mid \Psi(t) X(t) P_{2} X^{-1}(0)\left(\lambda_{n} X_{0}(0)\right)+ \\
& +\Psi(t) X(t)\left(I-P_{2}\right) X^{-1}(0) X_{0}(0)-\Psi(t) X(t) X^{-1}(0) X_{0}(0) \mid= \\
& =\left|\Psi(t) X(t) P_{2} X^{-1}(0)\left(\left(\lambda_{n}-1\right) X_{0}(0)\right)\right| \leq \\
& \leq\left|\lambda_{n}-1\right|\left|\Psi(t) X(t) P_{2}\right|\left|X^{-1}(0) X_{0}(0)\right| .
\end{aligned}
$$

Thus,

$$
\lim _{n \rightarrow \infty} \Psi(t) X_{n}(t)=\Psi(t) X_{0}(t) \text {, uniformly on } \mathbb{R}_{+} .
$$

It follows that the linear matrix differential equation (1.4) is $\Psi$ - conditionally stable on $\mathbb{R}_{+}$.

The proof is now complete.

Remark 3.1. Theorems 3.1 and 3.2 generalize a similar results in connection with the classical stability or $\Psi$ - conditional stability in [2], [3].

Sufficient conditions for $\Psi$ - conditional stability are given in the following theorems. At first, we consider the following hypotheses:

$\left(\mathbf{H}_{\mathbf{1}}\right)$ There exist supplementary projections $P_{i}: \mathbb{R}^{d} \longrightarrow \mathbb{R}^{d}, P_{1} \neq 0, P_{2} \neq 0$ and a constant $K>0$ such that the fundamental matrix $\mathrm{X}(\mathrm{t})$ for the linear matrix differential equation (1.4) satisfies one of following conditions:

i. $\left\{\begin{array}{l}\left|\Psi(t) X(t) P_{1} X^{-1}(s) \Psi^{-1}(s)\right| \leq K, \text { for } 0 \leq s \leq t \\ \left|\Psi(t) X(t) P_{2} X^{-1}(s) \Psi^{-1}(s)\right| \leq K, \text { for } 0 \leq t \leq s\end{array}\right.$;

ii. $\int_{0}^{t}\left|\Psi(t) X(t) P_{1} X^{-1}(s) \Psi^{-1}(s)\right| d s+$

$$
+\int_{t}^{\infty}\left|\Psi(t) X(t) P_{2} X^{-1}(s) \Psi^{-1}(s)\right| d s \leq K \text {, for } t \in \mathbb{R}_{+} .
$$

$\left(\mathbf{H}_{2}\right) \mathrm{A}_{1}(\mathrm{t})$ is a $d \times d$ continuous matrix function on $\mathbb{R}_{+}$that satisfies one of following conditions: 
i. $\mathrm{M}=\sup _{t \geq 0}\left|\Psi(t) A_{1}(t) \Psi^{-1}(t)\right|$ is a sufficiently small number;

ii. $\lim _{t \rightarrow \infty}\left|\Psi(t) A_{1}(t) \Psi^{-1}(t)\right|=0$;

iii. $\int_{0}^{\infty \rightarrow \infty}\left|\Psi(t) A_{1}(t) \Psi^{-1}(t)\right| d t$ is convergent.

Now, we have the following results:

Theorem 3.3. Suppose that:

1). the hypothesis $\left(\mathrm{H}_{1}-\mathrm{i}\right)$ holds;

$2)$. the hypothesis $\left(\mathrm{H}_{2}-\mathrm{iii}\right)$ holds;

3 ). the linear matrix differential equations (1.4) and (3.1) are $\Psi-$ unstable on $\mathbb{R}_{+}$.

Then, the linear matrix differential equation (3.1) is $\Psi$ - conditionally stable on $\mathbb{R}_{+}$.

Proof. It is similar with the proof of Theorem 3, [3].

Remark 3.2. This Theorem is the variant of Theorem 3, [3], for a linear matrix differential equation.

Theorem 3.4. Suppose that:

1). the hypotheses $\left(\mathrm{H}_{1}-\mathrm{i}\right.$, ii) hold;

2). the hypothesis $\left(\mathrm{H}_{2}-\mathrm{i}\right.$ or ii) holds;

2). the hypothesis $\left(\mathrm{H}_{2}-\mathrm{iii}\right)$ holds.

Then, the linear matrix differential equation (3.1) is $\Psi$ - conditionally stable on $\mathbb{R}_{+}$.

Proof. It results from Theorem above and Theorems 2, 3, [4], variant for a linear matrix differential equation.

Remark 3.3. The hypothesis $\left(\mathrm{H}_{1}\right)$ of the Theorems above certainly can be satisfied if $\mathrm{A}(\mathrm{t})=\mathrm{A}$ is a $d \times d$ real constant matrix which has characteristic roots with diferent real parts. Indeed, in this case, e.g., there exists an interval $(\alpha, \beta) \subset \mathbb{R}$ such that for $\lambda \in(\alpha, \beta), \Psi(t)=e^{-\lambda t} I_{d}$ and $X(t)=e^{t A}$ satisfy the inequalities

$$
\left\{\begin{array}{l}
\left|\Psi(t) X(t) P_{1} X^{-1}(s) \Psi^{-1}(s)\right| \leq K e^{(\alpha-\lambda)(t-s)}, \quad \text { for } 0 \leq s \leq t \\
\left|\Psi(t) X(t) P_{2} X^{-1}(s) \Psi^{-1}(s)\right| \leq K e^{(\beta-\lambda)(t-s)}, \text { for } 0 \leq t \leq s
\end{array},\right.
$$

where $P_{1} \neq 0, P_{2} \neq 0$ are supplementary projections.

There exists a similar situation if $\mathrm{A}(\mathrm{t})$ is a $d \times d$ real continuous periodic matrix (see Examples 1, 2, [3]).

Thus, the above results can be considered as a generalization of a well-known results in connection with the classical conditional stability (see e.g. [2])

Remark 3.4. If the linear equation (1.4) is only $\Psi$ - conditionally stable on $\mathbb{R}_{+}$(instead of the hypotheses $\left(\mathrm{H}_{1}-\mathrm{i}\right),\left(\mathrm{H}_{1}-\mathrm{ii}\right)$ ), then the perturbed equation (3.1) can't be $\Psi$ - conditionally stable on $\mathbb{R}_{+}$. This is shown by the Example 3, [3], in variant for a linear matrix differential equation. 


\section{$4 \Psi$ - conditional stability of linear Lyapunov matrix differential equations}

The purpose of this section is to study the $\Psi$ - conditional stability of the linear Lyapunov matrix differential equations (1.2) and (1.3).

Theorem 4.1. The linear Lyapunov matrix differential equations (1.3) is $\Psi$ - conditionally stable on $\mathbb{R}_{+}$if and only if the corresponding Kronecker product system (2.3) is $I_{d} \otimes \Psi$ - conditionally stable on $\mathbb{R}_{+}$.

Proof. It results from Lemma 2.7 and Remark 2.2.

The conditions for $\Psi$ - conditional stability of the linear Lyapunov matrix differential equation (1.3) can be expressed in terms of solutions or in terms of a fundamental matrices for (1.4) and (1.5).

Theorem 4.2. The linear Lyapunov matrix differential equation (1.3) is $\Psi-$ conditionally stable on $\mathbb{R}_{+}$if and only if it has a $\Psi$ - unbounded solution on $\mathbb{R}_{+}$and a nontrivial $\Psi$ - bounded solution on $\mathbb{R}_{+}$.

Proof. It results from Theorem 4.1, Theorem 3.1 (variant for systems), Lemmas 2.5 and 2.6.

Theorem 4.3. Let $\mathrm{X}(\mathrm{t})$ and $\mathrm{Y}(\mathrm{t})$ be fundamental matrices for the equations (1.4) and (1.5) respectively.

Suppose that:

a). There exists a projection $Q_{1}: \mathbb{R}^{d^{2}} \longrightarrow \mathbb{R}^{d^{2}}$ such that

$$
\left(Y^{T}(t) \otimes \Psi(t) X(t)\right) Q_{1}
$$

is unbounded on $\mathbb{R}_{+}$;

b). There exists a projection $Q_{2}: \mathbb{R}^{d^{2}} \longrightarrow \mathbb{R}^{d^{2}}, Q_{2} \neq 0$, such that

$$
\left(Y^{T}(t) \otimes \Psi(t) X(t)\right) Q_{2}
$$

is bounded on $\mathbb{R}_{+}$.

Then, the trivial solution of (1.3) is $\Psi$ - conditionally stable on $\mathbb{R}_{+}$.

Proof. It results from Theorem 4.1, Theorem 3.2 (variant for systems) and Lemmas 2.5, 2.6, 2.8.

Remark 4.1. It is easy to prove that the projection $Q_{1}$ have the form $Q_{1}=I_{1} \otimes P_{1}$, where $P_{1}: \mathbb{R}^{d} \longrightarrow \mathbb{R}^{d}$ is a projection (see Theorem 1, [5]).

For new results, we make the following hypotheses:

Let $\mathrm{X}(\mathrm{t})$ and $\mathrm{Y}(\mathrm{t})$ be fundamental matrices for the equations (1.4) and (1.5) respectively. 
$\left(\mathbf{H}_{\mathbf{3}}\right)$ There exist supplementary projections $P_{i}: \mathbb{R}^{d} \longrightarrow \mathbb{R}^{d}, P_{1} \neq 0, P_{2} \neq 0$ and the constants $K>0, L>0$, such that the fundamental matrices $\mathrm{X}(\mathrm{t})$ and $\mathrm{Y}(\mathrm{t})$ satisfy one of the following conditions:
a. $\left\{\begin{array}{l}\left|\Psi(t) X(t) P_{1} X^{-1}(s) \Psi^{-1}(s)\right| \leq K, \text { for } 0 \leq s \leq t \\ \left|\Psi(t) X(t) P_{2} X^{-1}(s) \Psi^{-1}(s)\right| \leq K, \text { for } 0 \leq t \leq s\end{array} ;\right.$
b. $\left\{\begin{array}{l}\left|\left(Y^{T}(t)\left(Y^{T}\right)^{-1}(s)\right) \otimes\left(\Psi(t) P_{1} \Psi^{-1}(s)\right)\right| \leq K, \text { for } 0 \leq s \leq t \\ \left|\left(Y^{T}(t)\left(Y^{T}\right)^{-1}(s)\right) \otimes\left(\Psi(t) P_{2} \Psi^{-1}(s)\right)\right| \leq K, \text { for } 0 \leq t \leq s\end{array} ;\right.$
c. $\begin{cases}\left|\left(Y^{T}(t)\left(Y^{T}\right)^{-1}(s)\right) \otimes\left(\Psi(t) X(t) P_{1} X^{-1}(s) \Psi^{-1}(s)\right)\right| \leq K, & \text { for } 0 \leq s \leq t \\ \left|\left(Y^{T}(t)\left(Y^{T}\right)^{-1}(s)\right) \otimes\left(\Psi(t) X(t) P_{2} X^{-1}(s) \Psi^{-1}(s)\right)\right| \leq K, & \text { for } 0 \leq t \leq s\end{cases}$
d. $\int_{0}^{t}\left|\left(Y^{T}(t)\left(Y^{T}\right)^{-1}(s)\right) \otimes\left(\Psi(t) X(t) P_{1} X^{-1}(s) \Psi^{-1}(s)\right)\right| d s+$ $+\int_{t}^{\infty}\left|\left(Y^{T}(t)\left(Y^{T}\right)^{-1}(s)\right) \otimes\left(\Psi(t) X(t) P_{2} X^{-1}(s) \Psi^{-1}(s)\right)\right| d s \leq L$, for $t \geq 0$.

$\left(\mathbf{H}_{\mathbf{4}}\right)$ The continuous matrices $\mathrm{A}(\mathrm{t})$ and $\mathrm{B}(\mathrm{t})$ satisfy the conditions

$\mathbf{a}_{1}$. $\sup _{t \geq 0}\left|\Psi(t) A(t) \Psi^{-1}(t)\right|$ is sufficiently small;

$\mathbf{a}_{2} \cdot \lim _{t \rightarrow \infty}\left|\Psi(t) A(t) \Psi^{-1}(t)\right|=0$

$\mathbf{a}_{3} \cdot \int_{0}^{\infty}\left|\Psi(t) A(t) \Psi^{-1}(t)\right| d t$ is convergent;

$\mathbf{b}_{1} \cdot \sup _{t \geq 0}|B(t)|$ is sufficiently small;

$\mathbf{b}_{2} \cdot \lim _{t \rightarrow \infty}|B(t)|=0$;

$\mathbf{b}_{3} \cdot \int_{0}^{\infty}|B(t)| d t$ is convergent.

$\left(\mathbf{H}_{\mathbf{5}}\right)$ There exist a projection $Q: \mathbb{R}^{d^{2}} \longrightarrow \mathbb{R}^{d^{2}}$ (or a projection $P: \mathbb{R}^{d} \longrightarrow$ $\mathbb{R}^{d}$, such that $\left.Q=I_{d} \otimes P\right)$ and a positive constant M such that

a. $\int_{t}^{\infty}\left|\left(I_{d} \otimes \Psi(t) X(t)\right) Q\left(I_{d} \otimes X^{-1}(s) \Psi^{-1}(s)\right)\right| d s \leq M$, for all $t \geq 0$, (or $\int_{t}^{\infty}\left|\Psi(t) X(t) P X^{-1}(s) \Psi^{-1}(s)\right| d s \leq M$, for all $t \geq 0$ );

b. $\int_{t}^{\infty}\left|\left(Y^{T}(t) \otimes \Psi(t)\right) Q\left(\left(Y^{T}\right)^{-1}(s) \otimes \Psi^{-1}(s)\right)\right| d s \leq M$, for all $t \geq 0$, (or $\int_{t}^{\infty}\left|\left(Y^{T}(t)\left(Y^{T}\right)^{-1}(s)\right) \otimes\left(\Psi(t) P \Psi^{-1}(s)\right)\right| d s \leq M$, for all $t \geq 0$ );

c. $\int_{t}^{\infty}\left|\left(Y^{T}(t)\left(Y^{T}\right)^{-1}(s)\right) \otimes\left(\Psi(t) X(t) P X^{-1}(s) \Psi^{-1}(s)\right)\right| d s \leq M$, for all $t \geq 0$.

Theorem 4.4. Suppose that are satisfied one of the following hypotheses:

I. $\left(\mathrm{H}_{3}-\mathrm{a}\right),\left(\mathrm{H}_{4}-\mathrm{b}_{3}\right)$ and the systems (1.3) and (1.4) are $\Psi-$ unstable on $\mathbb{R}_{+}$;

II. $\left(\mathrm{H}_{3}-\mathrm{b}\right),\left(\mathrm{H}_{4}-\mathrm{a}_{3}\right)$ and the systems (1.3) and (1.5) are $\Psi$ - unstable on $\mathbb{R}_{+}$.

Then, the linear Lyapunov matrix differential equation (1.3) is $\Psi$ - conditionally stable on $\mathbb{R}_{+}$.

Proof. From Theorem 4.1, we know that the equation (1.3) is $\Psi$ - conditionally stable on $\mathbb{R}_{+}$if and only if the corresponding Kronecker product system 
associated with (1.3), i.e. the system (2.3), is $I_{d} \otimes \Psi$ - conditionally stable on $\mathbb{R}_{+}$.

In case I, we apply Theorem 3.3 (variant for systems) to the system

$$
z^{\prime}=\left(I_{d} \otimes A(t)\right) z+\left(B^{T}(t) \otimes I_{d}\right) z .
$$

In case II, we apply Theorem 3.3 (variant for systems) to the system

$$
z^{\prime}=\left(B^{T}(t) \otimes I_{d}\right) z+\left(I_{d} \otimes A(t)\right) z .
$$

Thus, the system (2.3) is $I_{d} \otimes \Psi$ - conditionally stable on $\mathbb{R}_{+}$.

From Theorem 4.1, the linear Lyapunov matrix differential equation (1.3) is $\Psi$ - conditionally stable on $\mathbb{R}_{+}$.

The proof is now complete.

Remark 4.2. 1. The hypothesis $\left(H_{5}-\right.$ a) ensures that system (1.4) is $\Psi-$ unstable on $\mathbb{R}_{+}$;

2. The hypothesis $\left(H_{5}-b\right)$ ensures that system (1.5) is $\Psi$ - unstable on $\mathbb{R}_{+}$; 3. The hypothesis $\left(\mathrm{H}_{5}-\mathrm{c}\right)$ ensures that system $(1.3)$ is $\Psi$ - unstable on $\mathbb{R}_{+}$. (see Theorem 2, [5]).

Theorem 4.5. Suppose that are satisfied one of the following hypotheses:

I. $\left(\mathrm{H}_{3}-\mathrm{a}\right),\left(\mathrm{H}_{3}-\mathrm{d}\right),\left(\mathrm{H}_{4}-\mathrm{b}_{1}\right)$ or $\left(\mathrm{H}_{4}-\mathrm{b}_{2}\right),\left(\mathrm{H}_{4}-\mathrm{b}_{3}\right)$;

II. $\left(\mathrm{H}_{3}-\mathrm{b}\right),\left(\mathrm{H}_{3}-\mathrm{d}\right),\left(\mathrm{H}_{4}-\mathrm{a}_{1}\right)$ or $\left(\mathrm{H}_{4}-\mathrm{a}_{2}\right),\left(\mathrm{H}_{4}-\mathrm{a}_{3}\right)$.

Then, the linear Lyapunov matrix differential equation (1.3) is $\Psi$ - conditionally stable on $\mathbb{R}_{+}$.

Proof. It is similar with the proof of above Theorem, using Theorem 3.4.

Theorem 4.6. Suppose that are satisfied one of the following hypotheses:

I. $\left(\mathrm{H}_{3}-\mathrm{c}\right),\left(\mathrm{H}_{3}-\mathrm{d}\right)$;

II. $\mathrm{A}_{1}(\mathrm{t})$ and $\mathrm{B}_{1}(\mathrm{t})$ are continuous $d \times d$ matrices functions on $\mathbb{R}_{+}$and satisfy one of the following conditions:

i. $\mathrm{M}=\sup _{t \geq 0}\left|I_{d} \otimes \Psi(t) A_{1}(t) \Psi^{-1}(t)+B_{1}^{T}(t) \otimes I_{d}\right|<\frac{1}{L}$

ii. $\lim _{t \rightarrow \infty}\left|I_{d} \otimes \Psi(t) A_{1}(t) \Psi^{-1}(t)+B_{1}^{T}(t) \otimes I_{d}\right|=0$

and the condition

iii. $\int_{0}^{\infty}\left|I_{d} \otimes \Psi(t) A_{1}(t) \Psi^{-1}(t)+B_{1}^{T}(t) \otimes I_{d}\right|$ is convergent.

Then, the linear Lyapunov matrix differential equation (1.2) is $\Psi$ - conditionally stable on $\mathbb{R}_{+}$.

Proof. From Theorem 4.1, we know that the equation (1.2) is $\Psi$ - conditionally stable on $\mathbb{R}_{+}$if and only if the corresponding Kronecker product system associated with (1.2), i.e.

$$
z^{\prime}=\left[I_{d} \otimes\left(A(t)+A_{1}(t)\right)+\left(B(t)+B_{1}(t)\right)^{T} \otimes I_{d}\right] z
$$


or

$$
z^{\prime}=\left[I_{d} \otimes A(t)+B^{T}(t) \otimes I_{d}\right] z+\left[I_{d} \otimes A_{1}(t)+B_{1}^{T}(t) \otimes I_{d}\right] z,
$$

is $I_{d} \otimes \Psi$ - conditionally stable on $\mathbb{R}_{+}$.

From Lemma 2.8, we know that $U(t)=Y^{T}(t) \otimes X(t)$ is a fundamental matrix for the system

$$
z^{\prime}=\left(I_{d} \otimes A(t)+B^{T}(t) \otimes I_{d}\right) z .
$$

The hypotheses ensure, via Theorem 3, [3] and Theorem 3, [5], that the system (4.1) is $I_{d} \otimes \Psi$ - conditionally stable on $\mathbb{R}_{+}$.

From Theorem 4.1, the equation (1.2) is $\Psi$ - conditionally stable on $\mathbb{R}_{+}$.

The proof is now complete.

Remark 4.3. If the linear equation (1.3) is only $\Psi$ - conditionally stable on $\mathbb{R}_{+}$, then the perturbed equation (1.2) can't be $\Psi$ - conditionally stable on $\mathbb{R}_{+}$.

This is shown by the next Example, transformed after an equation due to $\mathrm{O}$. Perron [13].

Example 4.1. Consider the equation (1.3) with

$$
A(t)=\left(\begin{array}{cc}
\sin \ln (t+1)+\cos \ln (t+1)-2 a & 0 \\
0 & -a
\end{array}\right)
$$

and

$$
B(t)=\left(\begin{array}{cc}
\alpha & 0 \\
0 & \beta
\end{array}\right)
$$

where $a, \alpha, \beta \in \mathbb{R}$, such that $0<2 a<1+\alpha$ and $\alpha \leq 0, \beta>0$.

Then, a fundamental matrices for the homogeneous equations (1.4) and (1.5) are

$$
X(t)=\left(\begin{array}{cc}
e^{(t+1)(\sin \ln (t+1)-2 a)} & 0 \\
0 & e^{-a(t+1)}
\end{array}\right)
$$

and

$$
Y(t)=\left(\begin{array}{cc}
e^{\alpha(t+1)} & 0 \\
0 & e^{\beta(t+1)}
\end{array}\right)
$$

respectively.

Let $\Psi$ be the matrix

$$
\Psi(t)=\left(\begin{array}{cc}
1 & 0 \\
0 & e^{a(t+1)}
\end{array}\right) .
$$

From Lemma 2.8, the matrix 


$$
\left(Y^{T}(t) \otimes X(t)\right)=\left(\begin{array}{cccc}
u(t) & 0 & 0 & 0 \\
0 & e^{(-a+\alpha)(t+1)} & 0 & 0 \\
0 & 0 & v(t) & 0 \\
0 & 0 & 0 & e^{(-a+\beta)(t+1)}
\end{array}\right)
$$

where $u(t)=e^{(t+1)(\sin \ln (t+1)-2 a+\alpha)}, v(t)=e^{(t+1)(\sin \ln (t+1)-2 a+\beta)}$, is a fundamental matrix for the system (2.3), i.e. for the corresponding Kronecker product system associated with equation (1.3).

It is easy to see that $\left(I_{2} \otimes \Psi(t)\right)\left(Y^{T}(t) \otimes X(t)\right)$ is unbounded on $\mathbb{R}_{+}$.

From Theorem 1, [4], it follows that the system $(2.3)$ is $I_{2} \otimes \Psi-$ unstable on $\mathbb{R}_{+}$.

In addition, from Theorem 1, [3], the system (2.3) is $I_{2} \otimes \Psi-$ conditionally stable on $\mathbb{R}_{+}$.

From Theorem 4.1, it follows that the linear equation (1.3) is $\Psi$ - conditionally stable on $\mathbb{R}_{+}$.

Now, if we take

$$
A_{1}(t)=\left(\begin{array}{cc}
0 & b e^{-a(t+1)} \\
0 & 0
\end{array}\right)
$$

where $b \in \mathbb{R}, b \neq 0$, then, a fundamental matrix for the corresponding system (2.3) of perturbed equation (1.2) is

$$
Z_{0}(t)=\left(\begin{array}{cccc}
b u(t) \int_{1}^{t+1} e^{-s \sin \ln s} d s & u(t) & 0 & 0 \\
e^{(-a+\alpha)(t+1)} & 0 & 0 & 0 \\
0 & 0 & b v(t) \int_{1}^{t+1} e^{-s \sin \ln s} d s & v(t) \\
0 & 0 & e^{(-a+\beta)(t+1)} & 0
\end{array}\right)
$$

As in Example 3, [3], we have that the all columns of $\left(I_{2} \otimes \Psi(t)\right) Z_{0}(t)$ are unbounded on $\mathbb{R}_{+}$. From Theorem 4.1 and Theorem 1, [3], it follows that the perturbed equation (1.2) is not $\Psi$ - conditionally stable on $\mathbb{R}_{+}$.

Finally, we have

$$
\Psi(t) A_{1}(t) \Psi^{-1}(t)=\left(\begin{array}{cc}
0 & b e^{-2 a(t+1)} \\
0 & 0
\end{array}\right) .
$$

Thus, $A_{1}(t)$ and $B_{1}(t)=O_{2}$ satisfy the condition II of Theorem 4.6.

\section{$5 \Psi$ - conditional stability of nonlinear Lyapunov matrix differential equations}

The purpose of this section is to study the $\Psi$ - conditional stability of the nonlinear matrix differential equation (5.1) and of the nonlinear Lyapunov 
matrix differential equation (1.1).

Theorem 5.1. Suppose that:

1). There exist supplementary projections $P_{i}: \mathbb{R}^{d} \longrightarrow \mathbb{R}^{d}, P_{i} \neq 0$, and a constant $K>0$ such that the fundamental matrix $\mathrm{U}(\mathrm{t})$ for the linear matrix differential equation (1.4) satisfies the conditions

$$
\left\{\begin{array}{l}
\left|\Psi(t) U(t) P_{1} U^{-1}(s) \Psi^{-1}(s)\right| \leq K, \text { for } 0 \leq s \leq t \\
\left|\Psi(t) U(t) P_{2} U^{-1}(s) \Psi^{-1}(s)\right| \leq K, \text { for } 0 \leq t \leq s
\end{array}\right.
$$

2 ). The continuous function $F: \mathbb{R}_{+} \times \mathbb{M}_{d \times d} \longrightarrow \mathbb{M}_{d \times d}$ is such that

$$
\int_{0}^{\infty}|\Psi(t) F(t, 0)| d t<\infty
$$

and

$$
\left|\Psi(t)\left(F\left(t, X_{1}\right)-F\left(t, X_{2}\right)\right)\right| \leq \gamma(t)\left|\Psi(t)\left(X_{1}-X_{2}\right)\right|,
$$

for all $t \geq 0$ and $X_{1}, X_{2} \in \mathbb{M}_{d \times d}$, where $\gamma: \mathbb{R}_{+} \longrightarrow \mathbb{R}_{+}$is a continuous function such that

$$
\int_{0}^{\infty} \gamma(t) d t<\infty
$$

3). The equation (1.4) is $\Psi$ - unstable on $\mathbb{R}_{+}$.

4). All $\Psi$ - bounded solutions of the equation

$$
X^{\prime}=A(t) X+F(t, X)
$$

are $\Psi$ - unstable on $\mathbb{R}_{+}$.

Then, all $\Psi$ - bounded solutions of the equation (5.1) are $\Psi$ - conditionally stable on $\mathbb{R}_{+}$.

If, in addition, the continuous matrix $\mathrm{B}(\mathrm{t})$ is such that

$$
\int_{0}^{\infty}|B(t)| d t<\infty
$$

then, all $\Psi$ - bounded solutions of the the linear Lyapunov matrix differential equation (1.1) are $\Psi$ - conditionally stable on $\mathbb{R}_{+}$.

Proof. Choose $t_{0}>0$ so large that

$$
\theta=K \int_{t_{0}}^{\infty} \gamma(t) d t<1
$$

We put

$$
S=\left\{X: \mathbb{R}_{+} \longrightarrow \mathbb{M}_{d \times d} \mid X \text { is continuous and } \Psi-\text { bounded on }\left[t_{0} \cdot \infty\right)\right\} .
$$


Define on the set $\mathrm{S}$ a norm by

$$
\|X\|=\sup _{t \geq t_{0}}|\Psi(t) X(t)| .
$$

It is well-known that $(S,\|\cdot\|)$ is a Banach real space.

For $X \in S$, we define

$$
(T X)(t)=\int_{t_{0}}^{t} U(t) P_{1} U^{-1}(s) F(s, X(s)) d s-\int_{t}^{\infty} U(t) P_{2} U^{-1}(s) F(s, X(s)) d s
$$

for all $t \geq t_{0}$.

For $v \geq t \geq t_{0}$,

$$
\begin{aligned}
& \left|\Psi(t) \int_{t}^{v} U(t) P_{2} U^{-1}(s) F(s, X(s)) d s\right|= \\
& =\left|\int_{t}^{v} \Psi(t) U(t) P_{2} U^{-1}(s) \Psi^{-1}(s) \Psi(s) F(s, X(s)) d s\right| \leq \\
& \leq \int_{t}^{v}\left|\Psi(t) U(t) P_{2} U^{-1}(s) \Psi^{-1}(s)\right||\Psi(s) F(s, X(s))| d s \leq \\
& \leq K \int_{t}^{v}|\Psi(s) F(s, X(s))| d s= \\
& =K \int_{t}^{v}|\Psi(s)(F(s, X(s))-F(s, 0)+F(s, 0))| d s \leq \\
& \leq K \int_{t}^{v}[|\Psi(s)(F(s, X(s))-F(s, 0))|+|\Psi(s) F(s, 0)|] d s \leq \\
& \leq K\left[\int_{t}^{v} \gamma(s)|\Psi(s) X(s)| d s+\int_{t}^{v}|\Psi(s) F(s, 0)|\right] d s \leq \\
& \leq K\|X\| \int_{t}^{v} \gamma(s) d s+K \int_{t}^{v}|\Psi(s) F(s, 0)| d s .
\end{aligned}
$$

It follows that the integral

$$
\int_{t}^{\infty} U(t) P_{2} U^{-1}(s) F(s, X(s)) d s
$$

is convergent.

From hypotheses, $(T X)(t)$ exists for $t \geq t_{0}$ and is continuous differentiable.

For $X \in S$ and $t \geq t_{0}$,

$$
\begin{aligned}
& |\Psi(t)(T X)(t)|= \\
& =\mid \int_{t_{0}}^{t} \Psi(t) U(t) P_{1} U^{-1}(s) \Psi^{-1}(s) \Psi(s) F(s, X(s)) d s- \\
& -\int_{t}^{\infty} \Psi(t) U(t) P_{2} U^{-1}(s) \Psi^{-1}(s) \Psi(s) F(s, X(s)) d s \mid \leq \\
& \leq \int_{t_{0}}^{t}\left|\Psi(t) U(t) P_{1} U^{-1}(s) \Psi^{-1}(s)\right||\Psi(s) F(s, X(s))| d s+ \\
& +\int_{t}^{\infty}\left|\Psi(t) U(t) P_{2} U^{-1}(s) \Psi^{-1}(s)\right||\Psi(s) F(s, X(s)) d s| d s \leq \\
& \leq K\left[\int_{t_{0}}^{t}|\Psi(s)(F(s, X(s))-F(s, 0)+F(s, 0))| d s+\right. \\
& \left.+\int_{t}^{\infty}|\Psi(s)(F(s, X(s))-F(s, 0)+F(s, 0))| d s\right] \leq \\
& \leq K\left[\int_{t_{0}}^{t}(\gamma(s)|\Psi(s) X(s)|+|\Psi(s) F(s, 0)|) d s+\right. \\
& \left.+\int_{t}^{\infty}(\gamma(s)|\Psi(s) X(s)|+|\Psi(s) F(s, 0)|) d s\right] \leq
\end{aligned}
$$




$$
\leq K\left(\|X\| \int_{t_{0}}^{\infty} \gamma(s) d s+\int_{t_{0}}^{\infty}|\Psi(s) F(s, 0)| d s\right) .
$$

This shows that $T S \subset S$.

Moreover, for any two $\Psi$ - bounded continuous functions $X_{1}(t), X_{2}(t)$,

$$
\begin{aligned}
& \left|\Psi(t)\left(\left(T X_{1}\right)(t)-\left(T X_{2}\right)(t)\right)\right|= \\
& =\mid \int_{t_{0}}^{t} \Psi(t) U(t) P_{1} U^{-1}(s) \Psi^{-1}(s) \Psi(s)\left(F\left(s, X_{1}(s)\right)-F\left(s, X_{2}(s)\right)\right) d s- \\
& -\int_{t}^{\infty} \Psi(t) U(t) P_{2} U^{-1}(s) \Psi^{-1}(s) \Psi(s)\left(F\left(s, X_{1}(s)\right)-F\left(s, X_{2}(s)\right)\right) d s \mid \leq \\
& \leq \int_{t_{0}}^{t}\left|\Psi(t) U(t) P_{1} U^{-1}(s) \Psi^{-1}(s) \Psi(s)\left(F\left(s, X_{1}(s)\right)-F\left(s, X_{2}(s)\right)\right)\right| d s+ \\
& +\int_{t}^{\infty}\left|\Psi(t) U(t) P_{2} U^{-1}(s) \Psi^{-1}(s) \Psi(s)\left(F\left(s, X_{1}(s)\right)-F\left(s, X_{2}(s)\right)\right)\right| d s \leq \\
& \leq K \int_{t_{0}}^{\infty} \gamma(s)\left|\Psi(s)\left(X_{1}(s)-X_{2}(s)\right)\right| d s \leq \\
& \leq\left(K \int_{t_{0}}^{\infty} \gamma(s) d s\right)\left\|X_{1}-X_{2}\right\| .
\end{aligned}
$$

It follows that

$$
\left\|T X_{1}-T X_{2}\right\| \leq \theta\left\|X_{1}-X_{2}\right\| \text {, for all } X_{1}, X_{2} \in S .
$$

This shows that $\mathrm{T}$ is a contraction of the $\operatorname{Banach}$ space $(S,\|\cdot\|)$.

It follows by the contraction principle that for any $\Psi$ - bounded function $Y(t)$ on $\mathbb{R}_{+}$, the integral equation

$$
X=Y+T X
$$

has a unique solution $X \in S$.

Furthermore, by the definition of $\mathrm{T}, X(t)-Y(t)$ is continuous differentiable and

$$
(X(t)-Y(t))^{\prime}=A(t)(X(t)-Y(t))+F(t, X(t)), t \geq t_{0} .
$$

Hence, if $Y(t)$ is a $\Psi$ - bounded solution of (1.4), then the corresponding solution $X(t)$ of (5.2) is a $\Psi$ - bounded solution of (5.1). Conversely, if $X(t)$ is a $\Psi$ - bounded solution of (5.1), the function $Y(t)$ defined by (5.2) is a $\Psi$ - bounded solution of (1.4).

Thus, the equation (5.2) establishes a $1-1$ correspondence $\mathrm{C}$ between the $\Psi-$ bounded solutions of (1.4) and (5.1): $X=C Y$.

If we subtract from (5.2) the analogous equation $X_{0}=Y_{0}+T X_{0}$, we get $\left\|X-X_{0}\right\| \leq\left\|Y-Y_{0}\right\|+\left\|T X-T X_{0}\right\| \leq\left\|Y-Y_{0}\right\|+\theta\left\|X-X_{0}\right\|$, i.e.

$$
(1-\theta)\left\|X-X_{0}\right\| \leq\left\|Y-Y_{0}\right\|
$$

and

$\left\|Y-Y_{0}\right\| \leq\left\|X-X_{0}\right\|+\left\|T X-T X_{0}\right\| \leq(1+\theta)\left\|X-X_{0}\right\|$. 
Thus,

$$
(1+\theta)^{-1}\left\|Y-Y_{0}\right\| \leq\left\|X-X_{0}\right\| \leq(1-\theta)^{-1}\left\|Y-Y_{0}\right\| .
$$

This shows that the correspondence $\mathrm{C}$ is bicontinuous on the interval $\left[t_{0}, \infty\right)$. Since under the conditions of the theorem, the solutions of (1.4) and (5.1) are defined for all $t \geq 0$, are uniquely determined by their initial values and depend continuously on these initial values over any finite interval, we actually have continuity on the interval $[0, \infty)$.

F inally, let $X_{0}(t)$ be a $\Psi$ - bounded solution of (5.1). This solution is $\Psi-$ unstable on $\mathbb{R}_{+}$. Let $Y_{0}=X_{0}-T X_{0}$ be. From Theorem 3.1, the equation (1.4) is $\Psi$ - conditionally stable on $\mathbb{R}_{+}$. Thus, for the solution $Y_{0}$ of (1.4), there exists a sequence $\left(Y_{n}\right)$ of solutions of (1.4) defined on $\mathbb{R}_{+}$such that

$$
\lim _{n \rightarrow \infty} \Psi(t) Y_{n}(t)=\Psi(t) Y_{0}(t) \text {, uniformly on } \mathbb{R}_{+} .
$$

Let $X_{n}=C Y_{n}$ be. From (5.3), it follows that the sequence $\left(X_{n}\right)$ of solutions of (5.1), defined on $\mathbb{R}_{+}$, is such that

$$
\lim _{n \rightarrow \infty} \Psi(t) X_{n}(t)=\Psi(t) X_{0}(t), \text { uniformly on } \mathbb{R}_{+} .
$$

Thus, the $\Psi$ - bounded solution $X_{0}(t)$ of (5.1) is $\Psi$ - conditionally stable on $\mathbb{R}_{+}$.

The proof of the first part is complete.

The last part results from the above, if we put $X B(t)+F(t, X)$ instead of $F(t, X)$.

The proof is now complete.

Remark 5.1. The Theorem contains as a particular case a result concerning $\Psi$ - conditional stability of all $\Psi$ - bounded solutions of the differential system

$$
x^{\prime}=A(t) x+f(t, x) .
$$

Indeed, consider in (5.1)

$$
X=\left(\begin{array}{cccc}
x_{1} & x_{1} & \cdots & x_{1} \\
x_{2} & x_{2} & \cdots & x_{2} \\
\cdots & \cdots & \cdots & \cdots \\
x_{d} & x_{d} & \cdots & x_{d}
\end{array}\right)
$$

and

$$
F(t, X)=\left(\begin{array}{cccc}
f_{1}(t, x) & f_{1}(t, x) & \cdots & f_{1}(t, x) \\
f_{2}(t, x) & f_{2}(t, x) & \cdots & f_{2}(t, x) \\
\cdots & \cdots & \cdots & \cdots \\
f_{d}(t, x) & f_{d}(t, x) & \cdots & f_{d}(t, x)
\end{array}\right)
$$


where $x=\left(x_{1}, x_{2}, \cdots, x_{d}\right)^{T}$ and $f(t, x)=\left(f_{1}(t, x), f_{2}(t, x), \cdots, f_{d}(t, x)\right)^{T}$.

Now, the definitions and conditions for $\Psi$ - boundedness or $\Psi$ - conditional stability on $\mathbb{R}_{+}$of $x$ are the same for $\Psi$ - boundedness or $\Psi$ - conditional stability on $\mathbb{R}_{+}$of $X$.

We mention that in Theorem 5, [3], there exist a result concerning $\Psi$ - conditional stability of all $\Psi$ - bounded solutions of the nonlinear Volterra integrodifferential system

$$
x^{\prime}=A(t) x+\int_{0}^{t} F(t, s, x(s)) d s .
$$

Remark 5.2. In conditions of Theorem, in particular case $F(t, X)=X B(t)$, with $\int_{0}^{\infty}|B(t)| d t$ is convergent, the linear Lyapunov matrix differential equation (1.3) is $\Psi$ - conditionally stable on $\mathbb{R}_{+}$.

Corollary 5.1. Suppose that there exist supplementary projections $P_{i} \neq 0$, $P_{i}: \mathbb{R}^{d} \longrightarrow \mathbb{R}^{d}, i=1,2$ and a constant $K>0$ such that

$$
\left\{\begin{array}{l}
\left|\Psi(t) P_{1} \Psi^{-1}(s)\right| \leq K, \text { for } 0 \leq s \leq t \\
\left|\Psi(t) P_{2} \Psi^{-1}(s)\right| \leq K, \text { for } 0 \leq t \leq s
\end{array}\right.
$$

In addition, suppose that there exist a projection $P: \mathbb{R}^{d} \longrightarrow \mathbb{R}^{d}, P \neq 0$ and a constant $M>0$ such that

$$
\int_{t}^{\infty}\left|\Psi(t) P \Psi^{-1}(s)\right| d t \leq M, \text { for all } t \geq 0 .
$$

Let $F: \mathbb{R}_{+} \times \mathbb{M}_{d \times d} \longrightarrow \mathbb{M}_{d \times d}$ be a continuous function such that

$$
\int_{0}^{\infty}|\Psi(t) F(t, 0)| d t<\infty
$$

and

$$
\left|\Psi(t)\left(F\left(t, X_{1}\right)-F\left(t, X_{2}\right)\right)\right| \leq \gamma(t)\left|\Psi(t)\left(X_{1}-X_{2}\right)\right|,
$$

for all $t \geq 0$ and $X_{1}, X_{2} \in \mathbb{M}_{d \times d}$, where $\gamma: \mathbb{R}_{+} \longrightarrow \mathbb{R}_{+}$is a continuous function such that

$$
\int_{0}^{\infty} \gamma(t) d t<\infty
$$

Let $\mathrm{A}(\mathrm{t}), \mathrm{B}(\mathrm{t})$ be continuous matrix functions such that

$$
\int_{0}^{\infty}\left|\Psi(t) A(t) \Psi^{-1}(s)\right| d t<\infty
$$


and

$$
\int_{0}^{\infty}|B(t)| d t<\infty
$$

Suppose that all $\Psi$ - bounded solutions of the equation (1.1) are $\Psi$ - unstable on $\mathbb{R}_{+}$.

Then, all $\Psi$ - bounded solutions of the equation (1.1) are $\Psi$ - conditionally stable on $\mathbb{R}_{+}$.

Proof. It results from the above Theorem, if we take $\mathrm{O}_{2}$ instead of $\mathrm{A}(\mathrm{t})$ and $\mathrm{A}(\mathrm{t}) \mathrm{X}+\mathrm{XB}(\mathrm{t})+\mathrm{F}(\mathrm{t}, \mathrm{X})$ instead of $\mathrm{F}(\mathrm{t}, \mathrm{X})$.

Theorem 5.2. Suppose that:

1. There exist supplementary projections $P_{i}: \mathbb{R}^{d} \longrightarrow \mathbb{R}^{d}, P_{1} \neq 0, P_{2} \neq 0$ and a constant $K>0$ such that the fundamental matrices $\mathrm{X}(\mathrm{t})$ and $\mathrm{Y}(\mathrm{t})$ for the linear matrix differential equations (1.4) and (1.5) respectively satisfy the conditions

$$
\left\{\begin{array}{l}
\left|\left(Y^{T}(t)\left(Y^{T}\right)^{-1}(s)\right) \otimes\left(\Psi(t) X(t) P_{1} X^{-1}(s) \Psi^{-1}(s)\right)\right| \leq K, \quad \text { for } 0 \leq s \leq t \\
\left|\left(Y^{T}(t)\left(Y^{T}\right)^{-1}(s)\right) \otimes\left(\Psi(t) X(t) P_{2} X^{-1}(s) \Psi^{-1}(s)\right)\right| \leq K, \text { for } 0 \leq t \leq s
\end{array}\right.
$$

2. The continuous function $F: \mathbb{R}_{+} \times \mathbb{M}_{d \times d} \longrightarrow \mathbb{M}_{d \times d}$ is such that $F(t, 0)=0$ and satisfies the Lipschitz condition

$$
\left|\Psi(t)\left(F\left(t, X_{1}\right)-F\left(t, X_{2}\right)\right)\right| \leq \gamma(t)\left|\Psi(t)\left(X_{1}-X_{2}\right)\right|,
$$

for all $t \geq 0$ and $X_{1}, X_{2} \in \mathbb{M}_{d \times d}$, where $\gamma: \mathbb{R}_{+} \longrightarrow \mathbb{R}_{+}$is a continuous function such that

$$
\int_{0}^{\infty} \gamma(t) d t<\infty
$$

3. The linear Lyapunov matrix differential equation (1.3) is $\Psi$ - unstable on $\mathbb{R}_{+}$.

4. The trivial solution of the equation (1.1) is $\Psi$ - unstable on $\mathbb{R}_{+}$.

Then, the trivial solution of the equation (1.1) is $\Psi$ - conditionally stable on $\mathbb{R}_{+}$.

Proof. We will apply the Theorem 5.1, variant for differential system (see Remark 5.1).

From Lemma 2.7, we know that the trivial solution of the equation (1.1) is $\Psi$ - conditionally stable on $\mathbb{R}_{+}$if and only if the trivial solution of the corresponding Kronecker product system (2.1) is $I_{d} \otimes \Psi$ - conditionally stable on $\mathbb{R}_{+}$. 
From Lemma 2.8, we know that the matrix $Y^{T}(t) \otimes X(t)$ is a fundamental matrix for the linear homogeneous system associated with (2.1), i.e. for the differential system (2.3).

The hypothesis 1 ensures the hypothesis 1 of Theorem 5.1.

Let

$$
f(t, z)=\operatorname{Vec}(F(t, Z)), z=\operatorname{Vec}(Z),
$$

for $t \in \mathbb{R}_{+}$and $Z \in \mathbb{M}_{d \times d}$.

From hypothesis 2 and Lemma 2.6, it follows that

$$
\begin{aligned}
& \left\|\left(I_{d} \otimes \Psi(t)\right)\left(f\left(t, x_{1}\right)-f\left(t, x_{2}\right)\right)\right\|_{\mathbb{R}^{d^{2}}}= \\
& =\left\|\left(I_{d} \otimes \Psi(t)\right)\left(\operatorname{Vec}\left(F\left(t, X_{1}\right)\right)-\operatorname{Vec}\left(F\left(t, X_{2}\right)\right)\right)\right\|_{\mathbb{R}^{n^{2}}} \leq \\
& \leq\left|\Psi(t)\left(F\left(t, X_{1}\right)-F\left(t, X_{2}\right)\right)\right| \leq \gamma(t)\left|\Psi(t)\left(X_{1}-X_{2}\right)\right| \leq \\
& \leq d \gamma(t)\left\|\left(I_{d} \otimes \Psi(t)\right)\left(\operatorname{Vec}\left(X_{1}\right)-\operatorname{Vec}\left(X_{2}\right)\right)\right\|_{\mathbb{R}^{d^{2}}}= \\
& =d \gamma(t)\left\|\left(I_{d} \otimes \Psi(t)\right)\left(x_{1}-x_{2}\right)\right\|_{\mathbb{R}^{d^{2}}},
\end{aligned}
$$

for $t \geq 0$ and $x_{1}, x_{2} \in \mathbb{R}^{d^{2}}$.

Thus, it is ensured the hypothesis 2 of Theorem 5.1 .

In the end, the hypotheses 3 and 4 ensure the last hypotheses of Theorem 5.1 .

From Theorem 5.1, variant for differential system, the trivial solution of the system $(2.1)$ is $I_{d} \otimes \Psi-$ conditionally stable on $\mathbb{R}_{+}$. From Lemma 2.7 , results that the trivial solution of $(1.1)$ is $\Psi$ - conditionally stable on $\mathbb{R}_{+}$.

The proof is now complete.

Corollary 5.2. Suppose that:

1. There exist supplementary projections $P_{i}: \mathbb{R}^{d} \longrightarrow \mathbb{R}^{d}, P_{1} \neq 0, P_{2} \neq 0$ and a constant $K>0$ such that the fundamental matrices $\mathrm{X}(\mathrm{t})$ and $\mathrm{Y}(\mathrm{t})$ for the linear matrix differential equations (1.4) and (1.5) respectively satisfy the conditions:

$$
\begin{aligned}
& \text { i. }\left\{\begin{array}{l}
\left|\left(Y^{T}(t)\left(Y^{T}\right)^{-1}(s)\right) \otimes\left(\Psi(t) X(t) P_{1} X^{-1}(s) \Psi^{-1}(s)\right)\right| \leq K, \text { for } 0 \leq s \leq t \\
\left|\left(Y^{T}(t)\left(Y^{T}\right)^{-1}(s)\right) \otimes\left(\Psi(t) X(t) P_{2} X^{-1}(s) \Psi^{-1}(s)\right)\right| \leq K, \text { for } 0 \leq t \leq s
\end{array}\right. \\
& \text { ii. } \int_{0}^{t}\left|\left(Y^{T}(t)\left(Y^{T}\right)^{-1}(s)\right) \otimes\left(\Psi(t) X(t) P_{1} X^{-1}(s) \Psi^{-1}(s)\right)\right| d s+ \\
& \quad+\int_{t}^{\infty}\left|\left(Y^{T}(t)\left(Y^{T}\right)^{-1}(s)\right) \otimes\left(\Psi(t) X(t) P_{2} X^{-1}(s) \Psi^{-1}(s)\right)\right| d s \leq K \text {, for }
\end{aligned}
$$
$t \geq 0$.

2. The continuous function $F: \mathbb{R}_{+} \times \mathbb{M}_{d \times d} \longrightarrow \mathbb{M}_{d \times d}$ is such that $F(t, 0)=0$ and satisfies the Lipschitz condition

$$
\left|\Psi(t)\left(F\left(t, X_{1}\right)-F\left(t, X_{2}\right)\right)\right| \leq \gamma(t)\left|\Psi(t)\left(X_{1}-X_{2}\right)\right|,
$$


for all $t \geq 0$ and $X_{1}, X_{2} \in \mathbb{M}_{d \times d}$, where $\gamma: \mathbb{R}_{+} \longrightarrow \mathbb{R}_{+}$is a continuous function such that

$$
\int_{0}^{\infty} \gamma(t) d t<\infty
$$

and

$$
\Gamma=\sup _{t \geq 0} \gamma(t)<\frac{1}{K d}
$$

Then, the trivial solution of (1.1) is $\Psi$ - conditionally stable on $\mathbb{R}_{+}$.

Proof. It results from the above Theorem and Theorem 4, [5].

Corollary 5.3. Suppose that:

1. There exist supplementary projection $P_{i}: \mathbb{R}^{d} \longrightarrow \mathbb{R}^{d}, P_{1} \neq 0, P_{2} \neq 0$ and a constant $K>0$ such that the fundamental matrices $\mathrm{X}(\mathrm{t})$ and $\mathrm{Y}(\mathrm{t})$ for the linear matrix differential equations (1.4) and (1.5) respectively satisfy the conditions

$$
\left\{\begin{array}{l}
\left|\left(Y^{T}(t)\left(Y^{T}\right)^{-1}(s)\right) \otimes\left(\Psi(t) X(t) P_{1} X^{-1}(s) \Psi^{-1}(s)\right)\right| \leq K, \quad \text { for } 0 \leq s \leq t \\
\left|\left(Y^{T}(t)\left(Y^{T}\right)^{-1}(s)\right) \otimes\left(\Psi(t) X(t) P_{2} X^{-1}(s) \Psi^{-1}(s)\right)\right| \leq K, \text { for } 0 \leq t \leq s
\end{array}\right.
$$

2. There exist a projection $P: \mathbb{R}^{d} \longrightarrow \mathbb{R}^{d}, P \neq 0$ and a constant $M>0$ such that

$\int_{t}^{\infty}\left|\left(Y^{T}(t)\left(Y^{T}\right)^{-1}(s)\right) \otimes\left(\Psi(t) X(t) P X^{-1}(s) \Psi^{-1}(s)\right)\right| d s \leq K$, for all $t \geq 0$.

3. The continuous $d \times d$ matrices functions $A(t)$ and $B(t)$ satisfy the conditions

$$
\int_{0}^{\infty}\left|\Psi(t) A(t) \Psi^{-1}(t)\right| d t<\infty, \quad \int_{0}^{\infty}|B(t)| d t<\infty .
$$

Then, the equation (1.3) is $\Psi$ - conditionally stable on $\mathbb{R}_{+}$.

Proof. It results from the above Theorem and Theorem 2, [5].

Acknowledgements. This work was partially supported by the grant number $19 \mathrm{C} / 2014$, awarded in the internal grant competition of the University of Craiova.

\section{References}

[1] R. Bellman, Introduction to Matrix Analysis, McGraw-Hill Book Company, Inc. New York, 1960 
[2] W. A. Coppel, Stability and Asymptotic Behavior of Differential Equations, D. C. Heath and Company, Boston, 1965

[3] A. Diamandescu, On the $\Psi$ - conditional stability of the solutions of a nonlinear Volterra integro-differential system, Proceedings of the National Conference on Mathematical Analysis and Applications, (12-13 Dec. 2000), 89-106

[4] A. Diamandescu, On the $\Psi$-Instability of a Nonlinear Volterra Integro-Differential System, Bull. Math. Soc. Sc. Math. Roumanie, 46(94), No. 3-4, (2003), 103-119

[5] A. Diamandescu, On the $\Psi$ - instability of nonlinear Lyapunov matrix differential equations, Analele Universităţii de Vest, Timişoara, Seria Matematică - Informatică, XLIX, 1, (2011), 21-37

[6] A. Diamandescu, On $\Psi$ - stability of a nonlinear Lyapunov matrix differential equations, Electronic Journal of Qualitative Theory Differential Equations, 54, (2009), $1-18$

[7] A. Diamandescu, On $\Psi$-asymptotic stability of nonlinear Lyapunov matrix differential equations, Analele Universităţii de Vest, Timişoara, Seria Matematică - Informatică, L, 1, (2012), 3-25

[8] A. Diamandescu, On the $\Psi$ - strong stability of nonlinear Lyapunov matrix differential equations, to appear

[9] A. Diamandescu, On the $\Psi$ - Exponential Asymptotic Stability of Nonlinear Lyapunov Matrix Differential Equations, Analele Universităţii de Vest, Timişoara, Seria Matematică - Informatică, LI, 2, (2013), 7-28

[10] M.S.N. Murty and Suresh Kumar, On dichotomy and conditioning for twopoint boundary value problems associated with first order matrix Lyapunov systems, J. Korean Math. Soc., 45, no. 8, (2008), 1361-1378

[11] M.S.N. Murty and Suresh Kumar, On $\Psi$ - boundedness and $\Psi$ - stability of matrix Lyapunov systems, J. Appl. Math. Comput, 26, (2008), 67-84

[12] M.S.N. Murty, B. V. Apparao, and Suresh Kumar, Controllability, observability and realizability of matrix Lyapunov systems, Bull. Korean math. Soc., 43, no. 1, (2006), 149-159

[13] O. Perron, Die Stabilitätsfrage bei Differentialgleichungen, Math. Z., 32, (1930), $703-728$

Aurel Diamandescu

Department of Applied Mathematics, University of Craiova

Al. I. Cuza no 13, 200585-Craiova, Romania

E-mail: adiamandescu@central.ucv.ro

Received: 6.08.2014

Accepted: 20.09.2014 